\title{
Erratum to: Polysomnographic findings in Rett syndrome: a case-control study
}

\author{
Marco Carotenuto $\cdot$ Maria Esposito • \\ Alfredo D'Aniello • Carmen Donatella Rippa • \\ Francesco Precenzano • Antonio Pascotto • \\ Carmela Bravaccio • Maurizio Elia
}

Published online: 26 January 2013

(C) Springer-Verlag 2013

Erratum to: Sleep Breath

DOI 10.1007/s11325-012-0654-x

This erratum is published as the values of table 1 had various discrepancies in the article. Table 1 should now be read as:

The online version of the original article can be found at http://dx.doi.org/ 10.1007/s11325-012-0654-x.

M. Carotenuto $(\triangle) \cdot$ M. Esposito $\cdot$ A. D'Aniello $\cdot$ C. D. Rippa

F. Precenzano $\cdot$ A. Pascotto

Center for Sleep Disorders in Childhood-Clinic of Child and

Adolescent Neuropsychiatry, Second University of Naples,

Via Sergio Pansini n.5 PAD XI,

80131 Naples, Italy

e-mail: marco.carotenuto@unina2.it

C. Bravaccio

Department of Pediatries, Federico II University,

Naples, Italy

M. Elia

Oasi Institute for Research on Mental Retardation

and Brain Aging (IRCCS),

Troina, Italy 
Table 1 Comparison between sleep scoring parameters found in children with Rett syndrome and in control subjects

\begin{tabular}{|c|c|c|c|c|c|c|}
\hline & \multicolumn{2}{|l|}{ Rett, $N=3$} & \multicolumn{2}{|c|}{ Control, $N=40$} & \multicolumn{2}{|c|}{ Mann-Whitney } \\
\hline & Mean & SD & Mean & SD & $U$ & $p$ \\
\hline TIB (min) & 591.5231 & 63.10771 & 585.7750 & 83.61189 & 220.5000 & NS \\
\hline SPT (min) & 562.1154 & 64.63778 & 558.0750 & 85.45036 & 216.5000 & NS \\
\hline TST (min) & 502.1692 & 83.12539 & 531.0625 & 79.97076 & 215.0000 & NS \\
\hline SOL (min) & 15.4154 & 12.13005 & 19.7625 & 14.29721 & 215.5000 & NS \\
\hline FRL (min) & 147.9000 & 60.04176 & 120.3625 & 45.75968 & 184.0000 & NS \\
\hline $\mathrm{SS} / \mathrm{h}$ & 12.9385 & 2.99125 & 8.2200 & 3.51161 & 83.5000 & 0.0013 \\
\hline AWN/h & 6.3308 & 2.97220 & 1.8700 & 1.75969 & 49.5000 & 0.000000 \\
\hline SE $(\%)$ & 84.7245 & 8.69177 & 90.7300 & 5.14548 & 139.0000 & NS \\
\hline WASO (\%) & 13.2226 & 9.53861 & 4.6900 & 4.42550 & 98.0000 & 0.000998 \\
\hline S1 (\%) & 0.8796 & 1.38641 & 3.1175 & 3.82776 & 82.0000 & NS \\
\hline S2 (\%) & 30.1054 & 8.39295 & 45.4325 & 27.70823 & 97.0000 & NS \\
\hline SWS (\%) & 43.2735 & 13.69332 & 28.9800 & 10.02699 & 108.0000 & 0.003273 \\
\hline REM (\%) & 12.3989 & 7.71837 & 22.1650 & 5.55539 & 83.0000 & 0.000149 \\
\hline $\mathrm{OAI} / \mathrm{h}$ & 5.6685 & 4.42456 & 0.3480 & 0.26705 & 0.0000 & 0.000 \\
\hline $\mathrm{CAI} / \mathrm{h}$ & 7.7815 & 3.80737 & 0.3085 & 0.28396 & 0.0000 & 0.000 \\
\hline $\mathrm{HI} / \mathrm{h}$ & 7.7685 & 5.37938 & 0.3598 & 0.29043 & 0.0000 & 0.000 \\
\hline AHI & 16.7362 & 3.51547 & 0.9940 & 0.20460 & 0.0000 & 0.000 \\
\hline ODI & 13.0923 & 4.92341 & 0.4125 & 0.30650 & 0.0000 & 0.000 \\
\hline Mean Oxygen saturation (\%) & 93.6608 & 2.16734 & 96.6700 & 1.01000 & 53.5000 & 0.000 \\
\hline PLM\% $\%$ & 9.5185 & 1.89454 & 2.8100 & 1.09820 & 0.0000 & 0.000 \\
\hline
\end{tabular}

$T I B$, time in bed; SPT, sleep period time; TST, total sleep time; $S O L$, sleep onset latency; FRL, first REM sleep latency; $S S$, stage shifts; $A W N$, awakenings; $S E$, sleep efficiency; $W A S O$, wake time after sleep onset; $N 1$, sleep stage $1 ; N 2$, sleep stage $2 ; N 3$, slow-wave sleep; REM, rapid eye movement sleep percentage.

$O A I$, obstructive apnea index/h; $C A I$, central apnea index $/ \mathrm{h} ; H I$, hypopnea index $/ \mathrm{h} ; A H I$, apnea/hypopnea index (normal value $\leq 1 / \mathrm{h}$ ), and $O D I$, oxygen desaturation index (normal value $\leq 1 / \mathrm{h}$ ); $P L M$, periodic limb movements\% (normal value $\leq 5 \%$ ). $N S$, not significant.

*Bonferroni-corrected value. 